\title{
Influence of dance therapy on the Parkinson's disease affected upper limb biomechanics
}

\author{
Donatas Lukšys' ${ }^{1}$, Dalius Jatužis ${ }^{2}$, Rūta Kaladytė-Lokominienè $\dot{3}^{3}$, Ramunè Bunevičiūtė \\ Gabrielė Mickute் ${ }^{5}$, Alvydas Juocevičius ${ }^{6}$, Julius Griškevičius ${ }^{7}$ \\ 1,7 Vilnius Gediminas Technical University, Lithuania \\ 2, 3,4 Clinics of Neurology and Neurosurgery of Vilnius University Hospital Santariškių Klinikos, Lithuania \\ ${ }^{5,6}$ Centre of Rehabilitation and Physiotherapy of Vilnius University Hospital Santariškių Klinikos, \\ Lithuania \\ E-mails: ${ }^{1}$ donatas.luksys@vgtu.lt (corresponding author), ${ }^{2}$ dalius.jatuzis@santa.lt, \\ ${ }^{3}$ ruta.kaladyte-lokominiene@santa.lt, ${ }^{4}$ ramune.buneviciute@santa.lt, ${ }^{5}$ gabriele.mickute@santa.lt, \\ ${ }^{6}$ alvydas.juocevicius@santa.lt, ${ }^{7}$ julius.griskevicius@vgtu.lt \\ (Received 1 April 2016; accepted 18 May 2016)
}

\begin{abstract}
In this paper we investigate influence of the dance (lindy hop) therapy on the Parkinson's disease affected upper limb biomechanics. Wireless inertial sensors were used to measure acceleration and angular velocity during multi-joint movements of both upper limbs. In this research only wrist's pronation-supination movements in sagittal plane were analysed. Obtained results shows that dance therapy has a positive influence on improved biomechanics of upper limbs and general decrease of UPDRS score.
\end{abstract}

Keywords: Parkinson's disease, wireless inertial sensors, dance, upper limb.

\section{Introduction}

Parkinson's disease (PD) is neurodegenerative disorder, which affects part of central nervous system (CNS), which is responsible for the control of voluntary movements. It is second most common neurological disorder in Europe [1]. PD is characterized by resting tremor, bradykinesia, rigidity and postural instability. Patients with PD are applicable to various types of physical activity programs that improve motor function. Dance movement therapy is one of the possible activity programs and for patients with PD is among the most popular therapy programs [2], like tango dance, for example [3]. Dance therapy has a positive effect on psychologically and emotionally than regular exercise. Effect of dance therapy is usually evaluated using various scales. One of the most commonly applied is Unified Parkinson's Disease Rating scale (UPDRS), which allows estimate of motor and non - motor symptoms. For the quantitative assessment of the dance, various motion capture systems are used [4]. There are different temporal parameters that allow evaluating the movement. For example, acceleration zero crossing rate calculation performed for detecting the activities of daily living [5]. Average acceleration during the pronation-supination movement is calculated for UPDRS evaluation [6] or average movement time of rapid alternating movement [7].

Goal of this research is quantitatively evaluate therapeutic effect of dance therapy on the biomechanics of the upper limb using inertial sensors.

\section{Methods}

In cooperation with Vilnius University Hospital Santariškių Klinikos neurology and rehabilitation, physical and sports centres, 14 patients (mean age $\pm \mathrm{SD}=65.43 \pm 9.41$, mean UPDRS score $\pm \mathrm{SD}=47.8571 \pm 17.6890$ ) with PD (average stage of disease $\pm \mathrm{SD}=$ $2.0714 \pm 0.6157$ ) have been recruited for the modified lindy hop dance therapy. Comparing to other dance types, lindy hop dance was chosen, because this dance training is the simplest one. In total, subjects have had 22 dance lessons. Before each lesson 15 minutes of moderate exercise was performed and then 45 minutes of dancing.

(C) 2016 The Authors. Published by VGTU Press. This is an open-access article distributed under the terms of the Creative Commons Attribution License (CC-BY 4.0), which permits unrestricted use, distribution, and reproduction in any medium, provided the original author and source are credited. 
Six wireless inertial sensors (Shimmer, Dublin) having nine degrees of freedom (acceleration, angular velocity and magnetic heading in 3D) were attached to each patient's hand, forearm and arm. Sensors recorded data at $51.2 \mathrm{~Hz}$ sampling rate, which is sufficient to measure the movement. Each subject was asked to perform pronation-supination motion task.

MATLAB software was used for the calculation and analysis of wrist pronation - supination movement using following metrics: acceleration zero crossing rate and average acceleration about $y$-axis, average movement time. Statistical analysis of the metrics was performed using IMB's SPSS v22 software. A paired-samples t-test with significance level of $\alpha=0.05$ was used to compare calculation parameters before and after the dance therapy. The block diagram in figure 1 shows the general scheme of experiment and analysis.

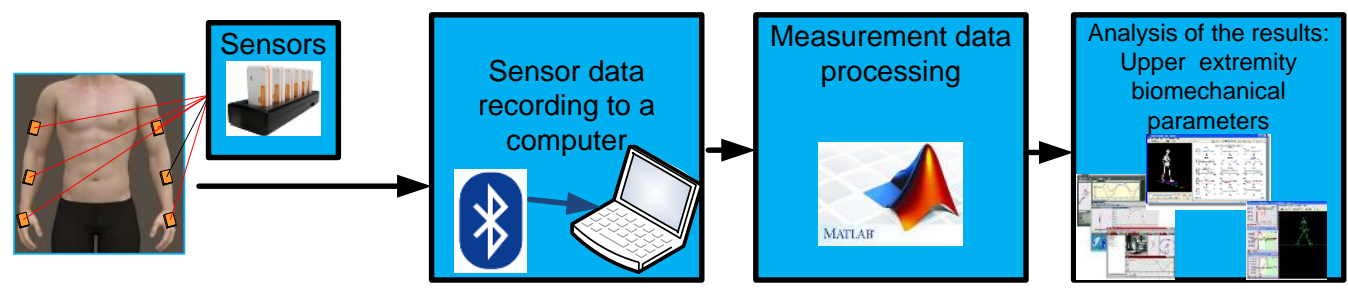

Fig. 1. Upper extremity motor biomechanics evaluation setup

\section{Results}

Calculation parameters are presented in Table 1. When analysing wrist pronation-supination movement, we found statistically significant difference of the right, left wrist average acceleration zero crossing rate, average movement time and average acceleration of the left wrist (bold values in the Table 1).

Table 1. Calculated parameters

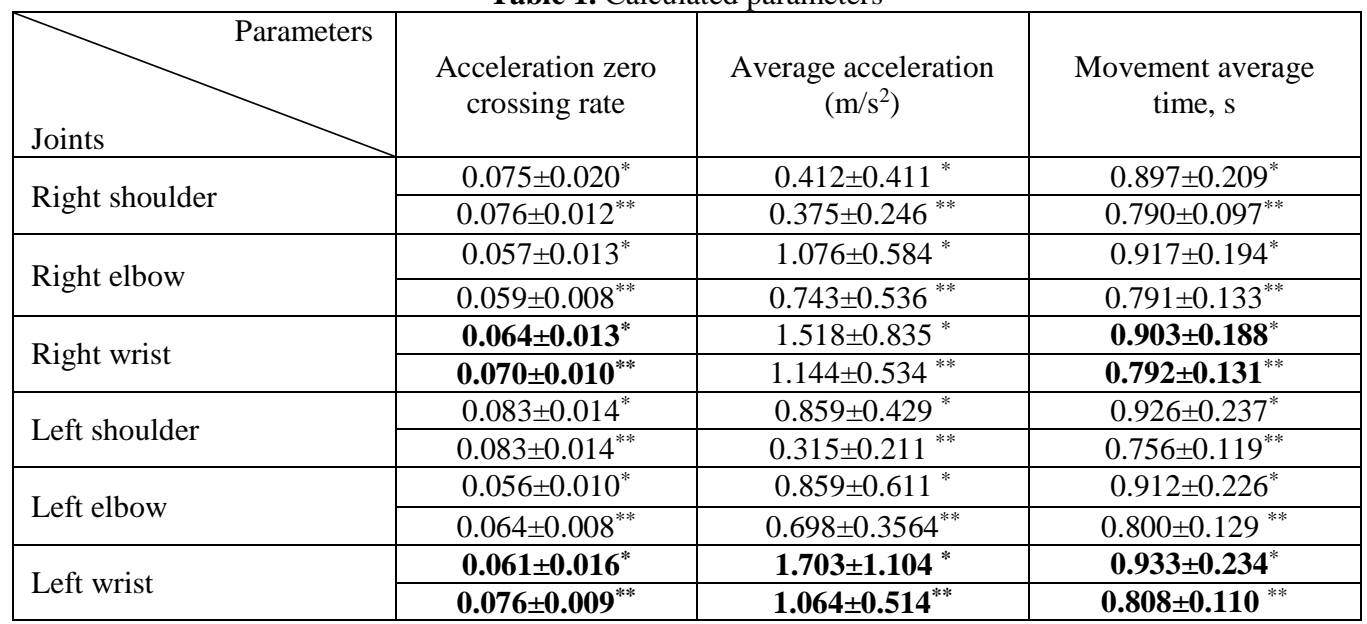

Note: $*$ - before (mean $\pm \mathrm{SD}), * *-\operatorname{after}($ mean $\pm \mathrm{SD})$

\section{Conclusions}

Comparing data before and after therapy program can be noted that dance therapy affects the upper limb movements. Zero crossing rate of the right wrist increases $(0.0636 \pm 0.0130$ to $0.0701 \pm 0.0104)$, and left wrist $(0.0608 \pm 0.0168$ to $0.0766 \pm 0.0091)$. Average acceleration values decrease in the left wrist $(1.7030 \pm 1.1043$ to $1.0640 \pm 0.5141)$. Movement average time right wrist 
decrease (of $0.9038 \pm 0.1884$ to $0.7927 \pm 0.1310$ ) and left wrist decrease (of $0.9331 \pm 0.2348$ to $0.8082 \pm 0.1107$ ). UPDRS score changed from $47.8571 \pm 17.6890$ to $40 \pm 13.8119$ after the dance therapy program. It can be said that the influence of dance for people with Parkinson's disease have a positive impact on upper limb biomechanics.

\section{References}

[1] de Dreu, M. J.; Kwakkel, G.; van Wegen, E. E. H. 2015. Partnered dancing to improve mobility for people with Parkinson's disease, Frontiers in Neuroscience 9: 444.

http://dx.doi.org/10.3389/fnins.2015.00444

[2] Chase, M. 2004. Marian Chace, her papers. Columbia: American Dance Therapy Association. 261 p.

[3] Hackney, M. E.; Earhart, G. M. 2009. Effects of dance on movement control in Parkinson's disease: a comparison of Argentine tango and American ballroom, Journal of Rehabilitation Medicine 41(6): 475-481. http://dx.doi.org/10.2340/16501977-0362

[4] Hackney, M. E.; Earhart, G. M. 2010. Effects of dance on balance and gait in severe Parkinson disease: a case study, Disability and Rehabilitation 32(8): 679-684.

http://dx.doi.org/10.3109/09638280903247905

[5] Olivares, A., et al. 2012. Detection of (in)activity periods in human body motion using inertial sensors: a comparative study, Sensors 12(5): 5791-5814. http://dx.doi.org/10.3390/s120505791

[6] Kaneko, M., et al. 2014. The comparison of pronation and supination between typically developing children and children with ADHD, Proceedings of the International MultiConference of Engineers and Computer Scientists IMECS 2014, March 12-14, 2014, Hong Kong, China. Hong Kong: Newswood Limited, 144-149.

[7] Soukoreff, R. W.; Zhao, J.; Ren, X. 2011. The entropy of a rapid aimed movement: Fitts' index of difficulty versus Shannon's entropy, in Proceedings of $13^{\text {th }}$ International Conference "Human Computer Interaction - INTERACT 2011”. September 5-9, 2011, Lisbon, Portugal. Berlin Heidelberg: Springer, 222-239. http://dx.doi.org/10.1007/978-3-642-23768-3_19 